\title{
Clinical and ECG patterns of pseudoinfarction in a young man with type 1 diabetes, diabetic ketoacidosis and normokalaemia
}

\section{ABSTRACT}

Diabetic ketoacidosis (DKA) can cause changes in the electrocardiogram (ECG) in the form of transient ST-segment depression, QT prolongation, changes in T-wave morphology and the appearance of $U$ wave, possibly due to changes in the serum potassium level. Occasional reports indicate the possibility of transient ST-segment elevation imitating myocardial infarction in the course of hyperkalaemia accompanying DKA. In this article we present a case of a 20-year-old male patient with type 1 diabetes mellitus, DKA and normokalaemia, who experienced severe retrosternal pain, and ECG presented ST-segment elevation imitating acute myocardial infarction of the anterior wall. On the basis of the performed cardiac tests, including laboratory testing, coronary angiography and ultrasound scan, acute coronary syndrome was ruled out. The regression of retrosternal pain and electrocardiographic changes with patient hydration and correction of metabolic disorders suggest the diagnosis of pseudopericarditis, i.e. non-infections irritation of the pericardial membranes due to the loss of fluid in the pericardial sac as a result of dehydration. The diagnosis of acute myocardial infarction based on ST-segment elevation in the ECG recording in a patient with diabetes mellitus and ketoacidosis, without concomitant hyperkalaemia, must be made very carefully, even in the presence of

Address for correspondence:

Piotr Molęda, M.D., PhD.

ul. Siedlecka 2, 72-010 Police

Phone/fax: +48 (91) 4253858

e-mail: pmoleda@wp.pl

Clinical Diabetology 2016, 5, 2, 73-76

DOI: $10.5603 /$ DK.2016.0012

Received: 03.04.2016

Accepted: 15.04.2016 retrosternal pain. The possibility of pseudopericarditis associated with severe dehydration must also be considered. (Clin Diabet 2016; 5, 2: 73-76)

Key words: diabetic ketoacidosis, type 1 diabetes mellitus, ST-segment elevation, normokalaemia, pseudopericarditis

\section{Introduction}

Diabetic ketoacidosis (DKA) is a severe acute metabolic complication associated with carbohydrate, fat and protein metabolism disorders, as well as impaired fluid-electrolyte and acid-base balance, as a result of severe insulin deficit [1]. The most common causes of DKA include: infection, discontinuation of or significant errors in insulin therapy, delay in diagnosis of diabetes mellitus, acute myocardial infarction, stroke, trauma, surgery, alcohol abuse, pregnancy.

Diabetic ketoacidosis can cause changes in the electrocardiogram (ECG). Most often they include transient ST-segment depression, QT prolongation, change in $T$ wave morphology and the appearance of $U$ waves [2]. The pathomechanism of these changes seems to be secondary to metabolic disorders, mainly related to the changes in serum potassium levels. ST-segment elevation in the course of DKA is extremely rare. There are only a few such reports in the available literature andthey mainly involved patients with hyperkalaemia [3-9]. In this article we present a patient with DKA, severe retrosternal pain and normokalaemia, in whom ST-segment elevation in the ECG was observed, imitating acute myocardial infarction. Cardiac diagnostics showed a normal coronary arteries imaging what ruled out the presence of ischaemia. 


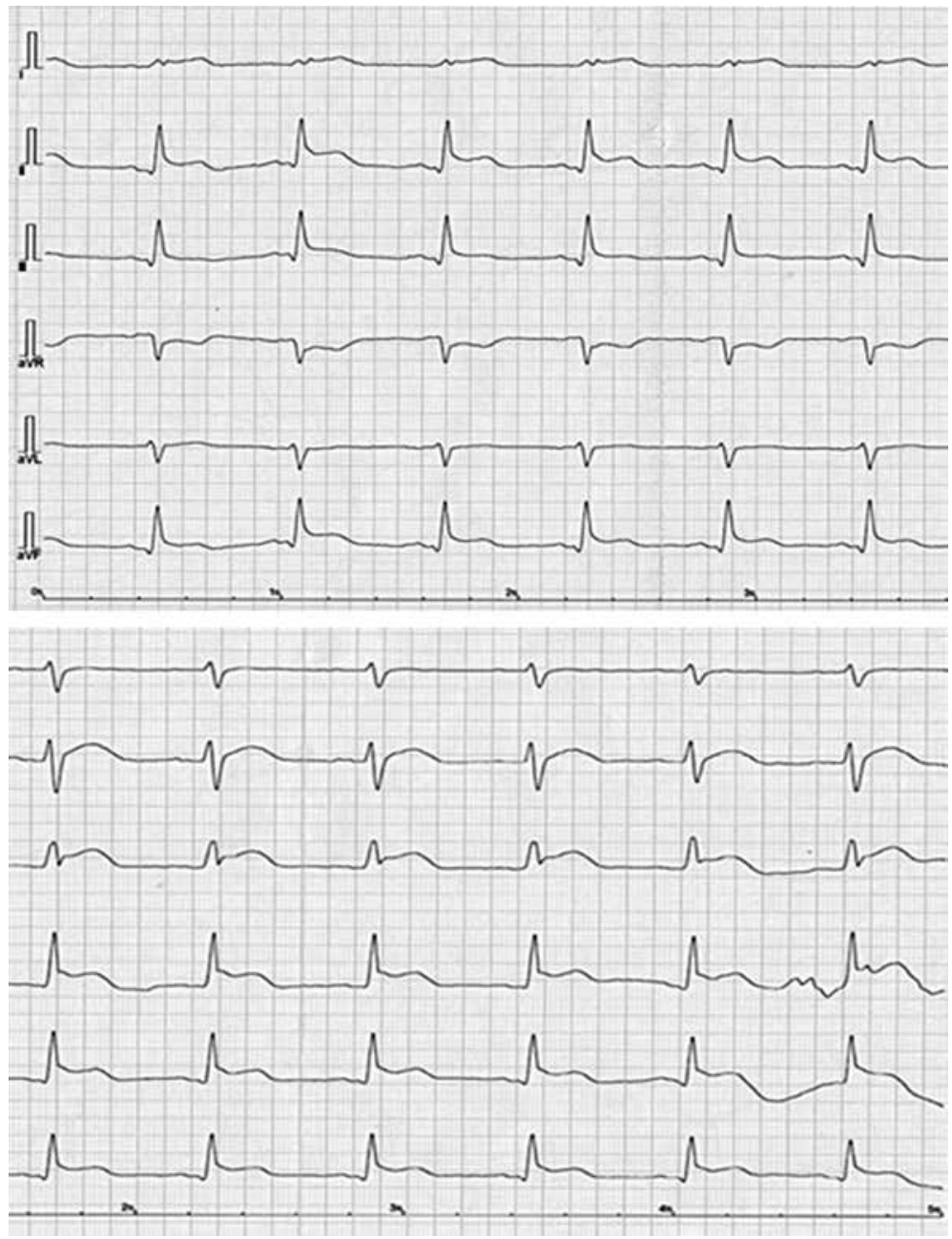

Figure 1. ST-segment elevation in the ECG

\section{Case study}

A 20-year-old male patient with type 1 diabetes mellitus diagnosed 10 years before, treated with a personal insulin pump with the use of rapid-acting insulin analogue, has been transported to the hospital due to weakness, low-grade fever, abdominal pain in the epigastrium and middle mesogastrium, nausea, vomiting as well as burning and squeezing retrosternal pain accompanied by high blood glucose values for 2 days. On admission, the patient was circulatory and respiratory stable, conscious, oriented, lethargic. On physical examination, acetone odour from the mouth, signs of significant dehydration, tachycardia (120 bpm), and abdominal tenderness in the middle mesogastrium were found. Based on the results of laboratory tests (hyperglycaemia, ketonuria, metabolic acidosis: $\mathrm{pH} 7.18, \mathrm{HCO}_{3} 5.6 \mathrm{mmol} / \mathrm{L}, \mathrm{BE}:-22.8 \mathrm{mmol} / \mathrm{L}$ ), diabetic ketoacidosis was diagnosed. Serum potassium concentration was $4.69 \mathrm{mmol} / \mathrm{L}$. Other laboratory results - Table 1 (examination 1). ECG showed sinus tachycardia $120 \mathrm{bpm}$ but no other abnormalities. The treatment administered involved fluids, electrolytes and insulin IV infusion.

In the subsequent hours, the patient reported worsening of retrosternal pain, weakness and shortness of breath. The ECG performed 10 hours after admission showed sinus tachycardia $120 \mathrm{bpm}, \mathrm{ST}$-segment elevation of $1 \mathrm{~mm}$ in the leads I, II, aVL, ST-segment elevation of $2 \mathrm{~mm}$ in the leads V2-V6, and ST-segment depression of $1 \mathrm{~mm}$ in the lead aVR. Markers of myocardial necrosis and serum potassium concentration were normal - Table 1 (examination 2).

Due to the nature of the ailments, acute coronary syndrome was suspected and the patient was referred to the Cardiology Department. The ECG performed 12 hours after the initial examination showed sinus tachycardia $106 \mathrm{bpm}, \mathrm{ST}$-segment elevation of $3 \mathrm{~mm}$ in leads I, II, aVL, aVF, V2-V6, and ST-segment depression of $2 \mathrm{~mm}$ in lead aVR (Figure 1). Markers of myocardial necrosis and serum potassium level were 
Table 1. Results of laboratory tests

\begin{tabular}{|c|c|c|c|c|}
\hline Parameters & $\begin{array}{c}\text { Examination } 1 \\
\text { (baseline) }\end{array}$ & $\begin{array}{c}\text { Examination } 2 \\
\text { (at } 10 \mathrm{~h})\end{array}$ & $\begin{array}{l}\text { Examination } 3 \\
\text { (at } 12 \text { and } 28 \mathrm{~h} \text { ) }\end{array}$ & $\begin{array}{l}\text { Examination } 4 \\
\text { (at } 52 \text { and } 76 \text { h) }\end{array}$ \\
\hline WBC $[\mathrm{G} / \mathrm{L}]$ & $45.78(3.8-10)$ & $34.13(3.8-10)$ & $33.42(4-10)$ & $7.72(3.8-10)$ \\
\hline Neutrophils (\%) & - & $86.7(46-75)$ & $85.7(46-75)$ & - \\
\hline $\mathrm{CRP}[\mathrm{mg} / \mathrm{L}]$ & $55.3(0-5)$ & $81.2(0-5)$ & $76.5 ; 63.6(0-5)$ & $16.8(0-5)$ \\
\hline Ketonuria & $(++++)$ & - & - & $(++++) ;(-)$ \\
\hline $\mathrm{pH}$ & $7.18(7.35-7.45)$ & $7.32(7.35-7.45)$ & $7.35(7.35-7.45)$ & $7.435(7.35-7.45)$ \\
\hline $\mathrm{HCO}_{3}[\mathrm{mmol} / \mathrm{L}]$ & $5.6(35-45)$ & $13.0(35-45)$ & $14.9(35-45)$ & $20.3(35-45)$ \\
\hline $\mathrm{BE}[\mathrm{mmol} / \mathrm{L}]$ & $-22.8(-3.0-3.0)$ & $-11.2(-3.0-3.0)$ & $-9.2(-3.0-3.0)$ & $-2.8(-3.0-3.0)$ \\
\hline Glucose [mmol/L] & $33.1(3.3-5.5)$ & $8.9(3.3-5.5)$ & $11.7(3.3-5.5)$ & $12.3(3.3-5.5)$ \\
\hline Creatinine $[\mu \mathrm{mol} / \mathrm{L}]$ & $177.7(61.9-106.1)$ & $100.8(61.9-106.1)$ & - & $76.9(61.9-106.1)$ \\
\hline $\mathrm{eGFR}\left[\mathrm{mL} / \mathrm{min} / 1.73 \mathrm{~m}^{2}\right]$ & $45.8(>90)$ & $88.1(>90)$ & - & $112.0(>90)$ \\
\hline Sodium [mmol/L] & $126(135-145)$ & $136(135-145)$ & $133 ; 131(136-145)$ & $139 ; 140$ (135-145) \\
\hline Potassium [mmol/L] & $4.69(3.5-5.5)$ & $4.06(3.5-5.5)$ & $4.0 ; 4.6(3.5-5.1)$ & $3.69 ; 4.49(3.5-5.5)$ \\
\hline Ionized calcium [mmol/L] & - & $0.91(1.12-1.32)$ & $1.25(1.15-1.3)$ & $1.43 ; 1.27(1.12-1.32)$ \\
\hline Inorganic phosphorus [mmol/L] & - & $1.1(0.81-1.45)$ & - & $0.64 ; 1.24(0.81-1.45)$ \\
\hline AST $[U / L]$ & - & $17(0-38)$ & - & - \\
\hline $\mathrm{ALT}[\mathrm{U} / \mathrm{L}]$ & - & $12(0-41)$ & - & - \\
\hline Creatine kinase $\mathrm{MB}[\mathrm{U} / \mathrm{L}]$ & - & $23.8(7-25)$ & $28 ; 18(1-24)$ & - \\
\hline \multirow[t]{2}{*}{ Troponin T } & - & $0.014 \mathrm{ng} / \mathrm{mL}(<0.014)$ & $<0.01 \mu \mathrm{g} / \mathrm{L}$ & - \\
\hline & & & $<0.01 \mu \mathrm{g} / \mathrm{L}$ & \\
\hline \multirow[t]{2}{*}{$\mathrm{HbA}_{1 \mathrm{c}}(\%)[\mathrm{mmol} / \mathrm{mol}]$} & - & - & - & $10.37(4.8-5.9)$ \\
\hline & & & & $90(29-41)$ \\
\hline
\end{tabular}

normal - Table 1 (examination 3). Echocardiography revealed no abnormalities in myocardial contractility or pericardial fluid. Coronary angiography imaging was normal. Due to the high glucose levels and sustained ketoacidosis, the patient was transferred back to the Diabetes Department.

On admission, the patient complained of mild retrosternal pain. Other than dry mucosae and tachycardia, physical examination revealed no abnormalities. The ECG showed sinus tachycardia $100 \mathrm{bpm}$ and abnormalities identical to those found previously. Serum potassium levels remained normal - Table 1 (examination 4). The following treatment was administered: fluids, electrolytes, insulin therapy, non-steroidal antiinflammatory drugs, antibiotics, proton pump inhibitor, obtaining glycaemic control, resolution of ketoacidosis, and after a few days resolution of retrosternal pain and ECG abnormalities. On the 11th day of treatment the patient was discharged home in good general condition.

\section{Discussion}

ST-segment elevation in diabetic ketoacidosis may suggest acute myocardial infarction. In the available literature there are a few reports of ST-segment elevation in patients with DKA, in whom acute coronary syndrome was ruled out on the basis of the performed cardiac diagnostics [3-10]. Hyperkalaemia was suggested as the cause of ST-segment elevation, as it changes the conduction of electrical impulses in the myocardium and the timing of the depolarisation period [3]. The effects of elevated serum potassium level on ECG recording in DKA are confirmed by the reports of regression of electrocardiographic abnormalities after achieving normokalaemia [4-8]. The persons reported in this literature are patients with type 1 diabetes mellitus, including three men aged 38-48 years, and two women aged 33 and 59 years, in whom transient ST-segment elevation was seen in ECG, imitating myocardial infarction of the anterior wall, and conduction disorders within the ventricles or the bundle branches, associated with hyperkalaemia $7.2-8.9 \mathrm{mmol} / \mathrm{L}$. The ECG abnormalities were reversible within a few hours after achieving normokalaemia. In all these cases patients denied the presence of chest pain.

Occasional reports indicate that significant pseudoinfarction ST-segment elevation in patients with DKA and significant hyperkalaemia $(7.7-8.3 \mathrm{mmol} / \mathrm{L})$ may not resolve immediately after lowering potassium levels to normal, but may persist for several days [3, 9]. In the reported cases of slightly longer persistence of ST-segment abnormalities, the patients also reported no chest pain. 
In the available literature there is only one report describing ST-segment elevation in a patient with DKA and normokalaemia [10]. It was a 58-year-old man with type 2 diabetes mellitus and DKA, who had serum potassium level of $4.4 \mathrm{mmol} / \mathrm{L}$ and ST-segment elevation in the leads II, III, and aVF. Also this patient reported no retrosternal pain. After 2 hours of treating diabetic ketoacidosis, ST-segment elevation subsided. Cardiac enzymes were normal. Echocardiography and coronary angiography showed no abnormalities.

The presented description of the 20-year-old patient with type 1 diabetes, ketoacidosis and normokalaemia is the second report suggesting that it is not hyperkalaemia but acidosis itself or other DKAspecific metabolic disorders that can cause changes in electrocardiographic image simulating recent myocardial infarction; this is, however, the first report with concomitant severe retrosternal pain. In the first place, we considered acute coronary syndrome as the cause of ST-segment elevation in this patient. This was suggested by the anginal nature of chest pain and ECG changes. Having ruled out acute coronary syndrome, acute pericarditis was considered as the possible cause of ST-segment elevation. This diagnosis was suggested by high WBC count and high blood levels of C-reactive protein, although in DKA patients inflammatory markers may be elevated without concomitant infection [11]. This patient, however, has no pericardial friction rub, ST-segment elevation was not accompanied by PQ-segment depression, and echocardiography showed no pericardial effusion. Other possible causes of ST-segment elevation in this patient include the toxic effect of ketoacidosis on intraventricular conduction or so-called pseudopericarditis, i.e. "non-infectious" irritation of the pericardial membranes caused by the loss of fluid from the pericardial sac as a result of dehydration. This phenomenon was described in 7 patients with diabetic ketoacidosis [12]. The above diagnosis is supported by regression of retrosternal pain and electrocardiographic changes following patient hydration and correction of metabolic disorders.
This clinical case clearly shows that the diagnosis of acute myocardial infarction based on ST-segment elevation in the ECG recording in a patient with diabetic ketoacidosis, without concomitant hyperkalaemia, must be made very carefully, even in the presence of retrosternal pain. The possibility of pseudopericarditis associated with severe dehydration must also be considered.

\section{Conflict of interest}

The authors have nothing to disclose.

\section{REFERENCES}

1. Umpierrez GE, Kitabchi AE. Diabetic ketoacidosis: risk factors and management strategies. Treat Endocrinol 2003; 2: 95-108.

2. Campbell IW, Duncan U, Clarke BF. Pericarditis in diabetic ketoacidosis. Br Heart J 1977; 39: 110-112.

3. Tatli E, Altun A, Yilmaztepe M. ST segment elevation following sinoventricular rhythm in a patient with diabetic ketoacidosis. Cardiol J 2007; 14: 497-499.

4. Moulik PK, Nethaji C, Khaleeli AA. Misleading electrocardiographic results in patients with hyperkalemia and diabetic ketoacidosis. BMJ 2002; 325: 1346-1347.

5. Ziakas A, Basagiannis C, Stiliadis I. Pseudoinfarction pattern in a patient with hyperkalemia, diabetic ketoacidosis and normal coronary vessels: a case report. J Med Case Rep 2010; 4: 115.

6. Wang K. Images in clinical medicine. "Pseudoinfarction" pattern due to hyperkalemia. N Engl J Med 2004, 5; 351: 593.

7. Cohen A, Utarnachitt RV. Electrocardiographic changes in a patients with hyperkalemia and diabetic acidosis associated with acute anteroseptal pseudomyocardial infarction and bifascicular block. Angiology 1981; 32: 361-364.

8. Lim YH, Anantharaman V. Pseudo myocardial infarct - electrocardiographic pattern in a patient with diabetic ketoacidosis. Singapore Med J 1998; 39: 504-506.

9. Chawla KK, Cruz J, Kramer NE, Towne WD. Electrocardiographic changes simulating acute myocardial infarction caused by hyperkalemia: report of a patient with normal coronary arteriograms. Am Heart K 1978; 95: 637-640.

10. Aksakal E, Duman H, Ulus T, Bayram E. Acute inferior pseudoinfarction pattern in a patient with normokalemia and diabetic ketoacidosis. Am J Emerg Med 2009; 27, 251: 3-5.

11. Karavanaki K, Kakleas K, Georga S et al. Plasma high sensitivity C-reactive protein and its relationship with cytokine levels in children with newly diagnosed type 1 diabetes and ketoacidosis. Clin Biochem 2012; 45: 1383-1388.

12. Benett KR, Blake TM. Pseudopericarditis in diabetic ketoacidosis. Southern Medical Journal 1971; 64: 610. 